\title{
Research Article Efficient TTI for 3G Multimedia Applications
}

\author{
Costas Chaikalis \\ Areos 28, 13121 Athens, Greece \\ Received 24 April 2007; Accepted 23 July 2007 \\ Recommended by Stavros Kotsopoulos
}

Time transmission interval (TTI) or outer block interleaving is an important task for the implementation of UMTS turbo coding in flat Rayleigh fading environment. An efficient TTI choice can save computational complexity. However, different multimedia scenarios are investigated using the maximum UMTS frame length, and simulation results are presented for the four possible outer block interleaver configurations in the case of flat Rayleigh fading channel. It is shown that different operating environments require an appropriate TTI in terms of bit error rate (BER) performance for the following data rates: $28.8 \mathrm{kbps}, 64 \mathrm{kbps}, 144 \mathrm{kbps}$, $384 \mathrm{kbps}$, and $2 \mathrm{Mbps}$.

Copyright ( 2007 Costas Chaikalis. This is an open access article distributed under the Creative Commons Attribution License, which permits unrestricted use, distribution, and reproduction in any medium, provided the original work is properly cited.

\section{INTRODUCTION}

Turbo coding [1] offers energy efficiencies close to the limits predicted by information theory with features that include parallel code concatenation, recursive convolutional encoding, interleaving, and an iterative decoding algorithm; while in fading environments, outer block interleaving should also be used. Soft-input/soft-output (SISO) decoder is the significant part of a turbo decoder: the concept of iterative decoding relies on the use of SISO decoders which calculate the a posteriori probabilities based on the received channel sequences and a priori information. One of the main candidate algorithms to be used in a SISO decoder is log maximum a posteriori (log-MAP) algorithm. A detailed description of $\log$-MAP is presented in [2-7], while turbo code basics and performance can be found in [7].

The European 3G standard is called Universal Mobile Telecommunications System (UMTS) and provides data rates up to $2 \mathrm{Mbps}$ giving the opportunity to mobile operators to offer multimedia applications to their customers, according to Table 1 . Turbo codes have been adopted as a channel coding scheme in UMTS for data rates higher than or equal to $28.8 \mathrm{kbps}[8,9]$.

The rest of the paper is organised as follows: Section 2 presents a short literature review on the area together with a brief description of UMTS data stream. Subsequently, Section 3 gives a description of the simulation environment used. The optimum outer block interleaver length is selected through simulation results and different implemen- tation scenarios in Section 4. Finally, after an efficient TTI selection according to simulation results, we conclude in Section 5.

\section{RELATED WORK AND UMTS DATA STREAM}

The investigation of multimedia applications over mobile cellular networks has been well addressed in published literature $[10,11]$, while different propagation (mobile channel) issues for such applications, tested by experimental procedures, have been presented in $[12,13]$.

In mobile communications, turbo codes provide very good coding gains in fading channels. Especially in frequency-flat Rayleigh fading channels, performance can be greatly improved if outer block interleaving is used $[7,14]$. This is because turbo encoding without outer block interleaving cannot correct the burst errors induced in a correlated fading channel, since they are more effective with random errors.

In [15], it is shown that the number of columns is the critical parameter in the design of outer block interleavers for turbo codes over such channels. The higher the mobile speed, the larger number of columns needed. In [16], simulation results are presented for the four different UMTS TTIs using flat Rayleigh fading and convolutional coding. Only the coding rate is varied for a terminal speed of $50 \mathrm{~km} / \mathrm{h}$, a frame length of 504 bits and a bit rate of $64 \mathrm{kbps}$. For these parameters and different signal-to-noise ratios (SNRs), a TTI of 80 milliseconds is shown to achieve the best FER 
TABLE 1: UMTS multimedia services.

\begin{tabular}{l|l}
\hline Category & Multimedia applications \\
\hline Entertainment & $\begin{array}{l}\text { Internet, video, text, picture, and } \\
\text { multimedia messaging, datacast, } \\
\text { personalisation applications (ring tone, } \\
\text { screen saver, desk top) }\end{array}$ \\
\hline Work & $\begin{array}{l}\text { Call with image and data stream, IP } \\
\text { telephony, notepad, 2-way video } \\
\text { conferencing, directory services, work group, } \\
\text { telepresence, FTP, instant voicemail, colour } \\
\text { fax }\end{array}$ \\
\hline Shopping & $\begin{array}{l}\text { E-commerce, e-cash, e-wallet, credit card, } \\
\text { telebanking }\end{array}$ \\
\hline Education & $\begin{array}{l}\text { Online libraries, search engines, remote } \\
\text { attendance, field research }\end{array}$ \\
\hline Health & $\begin{array}{l}\text { Telemedicine, remote diagnose, and health } \\
\text { monitoring }\end{array}$ \\
\hline Automation & $\begin{array}{l}\text { Home automation, traffic telematics, machine, } \\
\text { telemetry }\end{array}$ \\
\hline services & $\begin{array}{l}\text { Location sensitive information and } \\
\text { guidance, e-tour, location awareness, time } \\
\text { tables, e-ticketing }\end{array}$ \\
\hline $\begin{array}{l}\text { TV, radio, PC, access to remote computer, } \\
\text { MP3 player, camera, GPS, remote control } \\
\text { unit }\end{array}$ \\
\hline trional
\end{tabular}

performance. Similar to the approach described in [16], but for turbo codes, in this paper, we discuss the effect of outer block interleaving on UMTS turbo codes performance for a constant frame length, log-MAP algorithm for turbo decoder, five different bit rates, and also different operating environments.

In [17], a reconfigurable block interleaver for block codes over flat Rayleigh fading is presented. A formula is derived to calculate the interleaving depth according to the Doppler frequency. It is also mentioned that the bit error rate (BER) will be higher unless the right number of columns is employed. In this paper, we will show that the same conclusion is also drawn for turbo codes. Finally, in [5], we discuss different UMTS implementation scenarios for an outer block interleaver, different frame lengths, operating environments, and a constant bit rate. This paper considers the same approach but constant frame length and different bit rates.

UMTS data stream is described in $[8,9]$. Thus, a UMTS transport channel transfers the information over the radio interface from the medium access control sublayer of layer 2 to the physical layer. The characteristics of a transport channel are determined by its transport format set, which consists of different transport formats. The transport formats must have the same type of channel coding and TTI, while the transport block set or data frame size can vary. The transport block set determines the number of input bits to the channel encoder and can be transmitted every TTI, with possible values for TTI of 10, 20, 40, and 80 milliseconds. Every trans-
TABLE 2: Implementation scenarios, corresponding bit rates, and multimedia examples.

\begin{tabular}{l|c|c|l|l|c}
\hline \multirow{2}{*}{} & \multicolumn{5}{|c}{ Implementation scenario } \\
\cline { 2 - 6 } & 1 & 2 & 3 & 4 & 5 \\
\hline Bit rate $R_{b}$ & $28.8 \mathrm{kbps}$ & $64 \mathrm{kbps}$ & $144 \mathrm{kbps}$ & $384 \mathrm{kbps}$ & $2 \mathrm{Mbps}$ \\
\hline $\begin{array}{l}\text { Multimedia } \\
\text { application }\end{array}$ & $\begin{array}{l}\text { Fax } \\
\text { service }\end{array}$ & $\begin{array}{l}\text { Packet } \\
\text { data } \\
\text { services }\end{array}$ & $\begin{array}{l}\text { Packet } \\
\text { data } \\
\text { services }\end{array}$ & $\begin{array}{l}\text { Packet } \\
\text { data } \\
\text { services }\end{array}$ & $\begin{array}{l}\text { Packet } \\
\text { data } \\
\text { services }\end{array}$ \\
\hline
\end{tabular}

port channel is also assigned to a radio access bearer with a particular data rate.

\section{SIMULATION ENVIRONMENT}

A flat Rayleigh fading channel is described by

$$
y_{k}=\alpha_{k} \cdot x_{k}+n_{k}
$$

where $k$ is an integer symbol index, $x_{k}$ is a binary phase shift keying (BPSK) symbol amplitude $( \pm 1), n_{k}$ is a Gaussian random variable, and $y_{k}$ is a noisy received symbol. The fading amplitude $a_{k}$ is a sample from a correlated Gaussian random process with zero mean and is generated using the sum of sines or Jakes model [18].

In our simulations, a carrier frequency $f_{c}=2 \mathrm{GHZ}$ is considered. It is also assumed that 1000000 information bits are transmitted and grouped into frames whose length must be $\geq 40$ and $\leq 5114$, according to UMTS specifications [ 19 , 20]. For a particular transport channel, every TTI, the data with the characteristics specified in a transport format of the transport channel, is turbo-encoded at the transmitter. After turbo encoding and block interleaving, the bits are BPSKmodulated and transmitted through the mobile channel.

At the receiver, outer block deinterleaving and turbo decoding is performed. Floating point arithmetic is also used, while the receiver is assumed to have exact estimates of the fading amplitudes (perfect channel estimation without side information). The iterations of the turbo decoder are assumed to be eight.

\section{EFFICIENT TTI SELECTION}

The frame length used in our simulations is chosen to be the maximum length specified for UMTS, 5114 bits. Five typical input bit rates $R_{b}$ are considered according to UMTS specifications: $28.8 \mathrm{kbps}, 64 \mathrm{kbps}, 144 \mathrm{kbps}, 384 \mathrm{kbps}$, and $2 \mathrm{Mbps}$ [21]. These bit rates represent five different UMTS implementation scenarios, as Table 2 illustrates. Particularly, according to [21], scenario 1 can be applied to fax service, while scenarios 2, 3, 4, and 5 can be applied to any multimedia packet data service like conventional internet services (webbrowsing, electronic mail, file transfer, video/audio streaming, e-commerce, videoconference), interactive data or combination with location information and mobility (locationbased services, navigation).

Furthermore, four different mobile speeds are considered for each implementation scenario: $4 \mathrm{~km} / \mathrm{h}$ (corresponds 
TABLE 3: Normalised fade rates for different Doppler frequencies, mobile terminal speeds, and implementation scenarios.

\begin{tabular}{|c|c|c|c|c|c|c|}
\hline \multirow{2}{*}{$\begin{array}{l}\text { Terminal } \\
\text { speed }(\mathrm{km} / \mathrm{h})\end{array}$} & \multirow{2}{*}{$\begin{array}{l}\text { Doppler freq. } \\
f_{d}(\mathrm{~Hz})\end{array}$} & \multicolumn{5}{|c|}{ Scenario } \\
\hline & & 1 & 2 & 3 & 4 & 5 \\
\hline 4 & 7.4 & 0.000085 & 0.000038 & 0.000017 & 0.0000064 & 0.0000012 \\
\hline 50 & 92.5 & 0.00107 & 0.00048 & 0.00021 & 0.00008 & - \\
\hline 100 & 185.1 & 0.0021 & 0.00096 & 0.00042 & 0.00016 & - \\
\hline 300 & 555.5 & 0.0064 & 0.0028 & 0.00128 & - & - \\
\hline
\end{tabular}

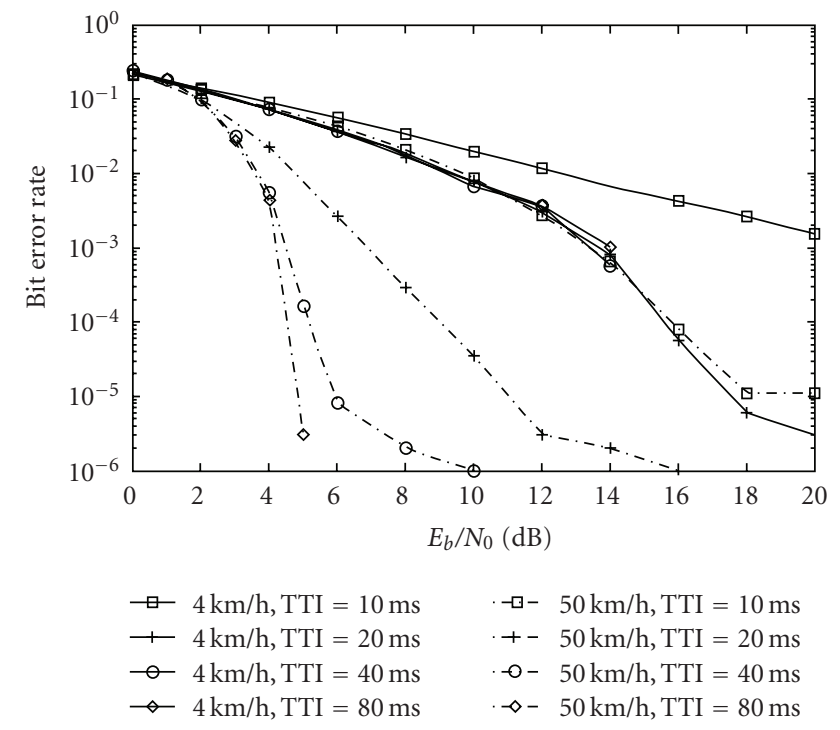

Figure 1: BER versus SNR for scenario 1 and $f_{d} T_{S}$ values 0.000085 and 0.00107 .

to indoor or low-range outdoor operating environment), 50 and $100 \mathrm{~km} / \mathrm{h}$ (urban or suburban outdoor operating environment), and $300 \mathrm{~km} / \mathrm{h}$ (rural outdoor operating environment). In Table 3, the different values of normalised fade rate $f_{d} T_{S}$ are presented for each scenario and terminal speed. For the first 3 scenarios (data rates $28.8 \mathrm{kbps}$, $64 \mathrm{kbps}$, and $144 \mathrm{kbps}$ ), all 4 mobile terminal speeds can be applied according to [22], whereas for scenarios 4 and 5, there are some limitations. Thus, according to [22], for scenario 4 (data rate $384 \mathrm{kbps}$ ), the mobile terminal speed of $300 \mathrm{~km} / \mathrm{h}$ cannot be considered, and for scenario 5 (data rate $2 \mathrm{Mbps}$ ), the mobile terminal speeds of 50,100, and $300 \mathrm{~km} / \mathrm{h}$ cannot be considered as well.

A scaling factor $s=0.7$ is also applied in log-MAP turbo decoding algorithm in our simulation model because, according to [6], $s=0.7$ and $s=0.8$ give the best performance improvement for log MAP in a flat-fading channel. In the following sections, BER performance is evaluated for each scenario and our goal is to find the optimum TTI value.

\subsection{Scenario 1 : bit rate $28.8 \mathrm{kbps}$}

In Figure 1, the BER performance of the simulated system for mobile speeds 4 and $50 \mathrm{~km} / \mathrm{h}$ is presented. For $4 \mathrm{~km} / \mathrm{h}$ and at a BER of $2 \times 10^{-3}$, there is a gain of approximately $6 \mathrm{~dB}$ for a TTI transition from 10 to 20 milliseconds. Thus,

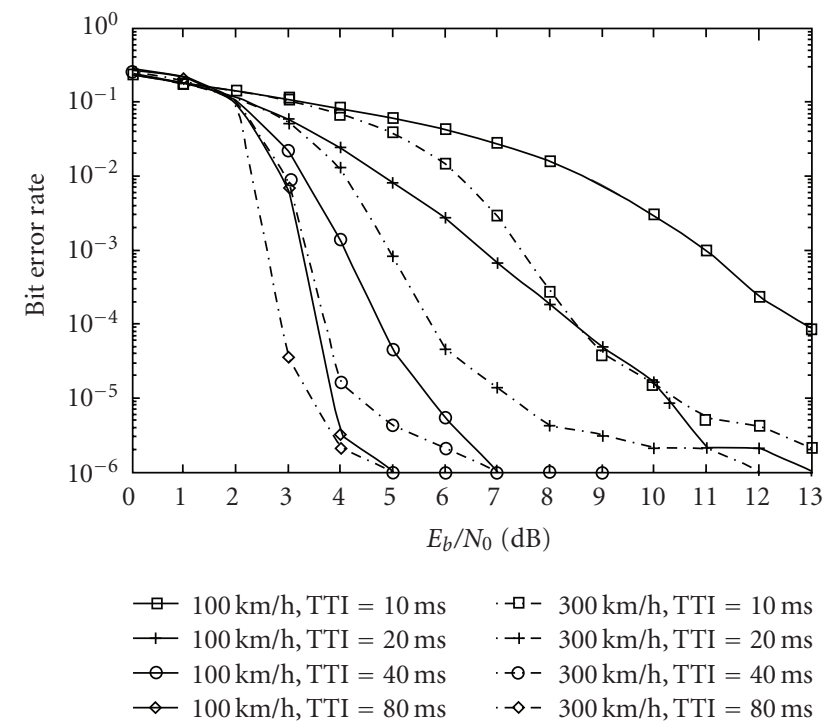

FIgURE 2: BER versus SNR for scenario 1 and $f_{d} T_{S}$ values 0.0021 and 0.0064 .

for $4 \mathrm{~km} / \mathrm{h}$, the optimum TTI value is 20 milliseconds: there is no BER improvement for larger TTI values. On the contrary, as Figure 1 shows, BER becomes worse for values larger than the optimum value of 20 milliseconds. Additionally, at a BER of $10^{-3}, 0.5 \mathrm{~dB}$ performance loss can be observed for 80 milliseconds compared to the optimum TTI value of 20 milliseconds. For $50 \mathrm{~km} / \mathrm{h}$ and at a BER of $10^{-3}$, there is a gain of approximately $6 \mathrm{~dB}$ for a TTI transition from 10 to 20 milliseconds. The gain decreases to $2 \mathrm{~dB}$ and $0.5 \mathrm{~dB}$ for a TTI transition from 20 milliseconds to 40 milliseconds and from 40 milliseconds to 80 milliseconds, respectively. Thus, for $50 \mathrm{~km} / \mathrm{h}$, the optimum TTI value is 80 milliseconds.

For Figure 2, the mobile terminal speeds are 100 and $300 \mathrm{~km} / \mathrm{h}$. Here, as Figure 2 shows, the optimum TTI value for both terminal speeds is 80 milliseconds. However, at a BER of $10^{-3}$ for $100 \mathrm{~km} / \mathrm{h}$, a gain of $4 \mathrm{~dB}, 2.5 \mathrm{~dB}$ and $1 \mathrm{~dB}$ is seen for a TTI transition from 10 to 20,20 to 40 , and 40 to 80 milliseconds, respectively. At the same BER for $300 \mathrm{~km} / \mathrm{h}$ terminal speed, a TTI increase from 10 to 20, 20 to 40 , and 40 to 80 milliseconds gives performance gains of $2.5 \mathrm{~dB}, 1.5 \mathrm{~dB}$, and $0.8 \mathrm{~dB}$, respectively.

\subsection{Scenario 2: bit rate 64 kbps}

Figure 3 presents the performance for the four different TTI values considering terminal speeds of $4 \mathrm{~km} / \mathrm{h}$ and $50 \mathrm{~km} / \mathrm{h}$. 


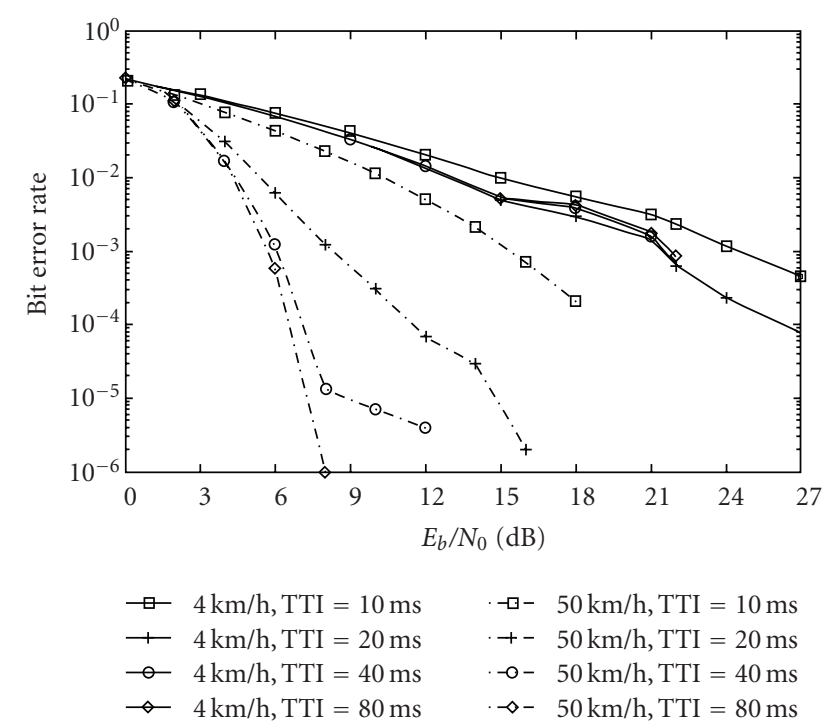

Figure 3: BER versus SNR for scenario 2 and $f_{d} T_{S}$ values 0.000038 and 0.00048 .

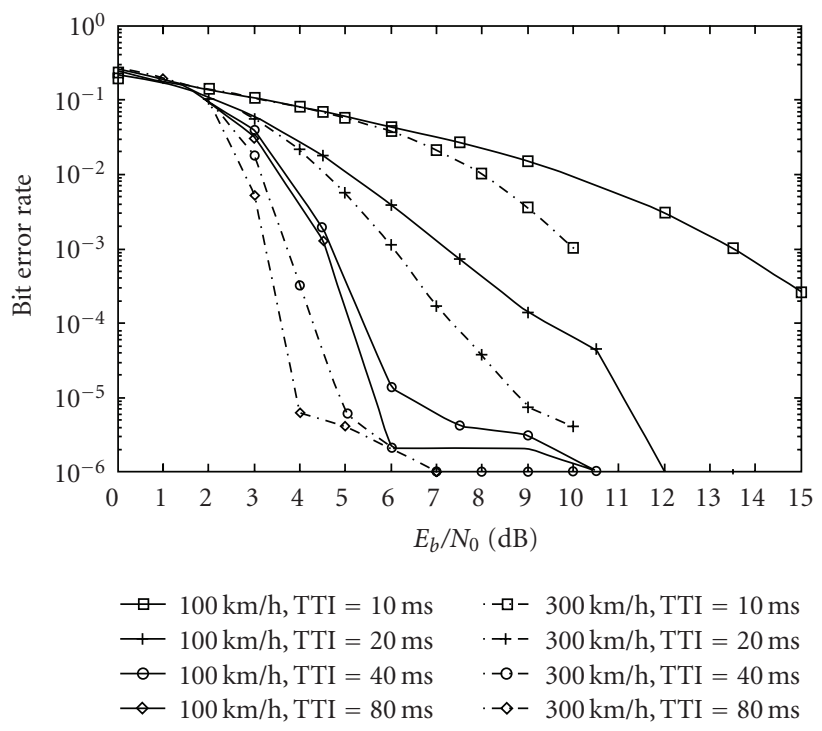

FIgURE 4: BER versus SNR for scenario 2 and $f_{d} T_{S}$ values 0.00096 and 0.0028 .

At a BER of $10^{-3}$ for $4 \mathrm{~km} / \mathrm{h}$, we see that a TTI increase from 10 to 20 milliseconds gives $4 \mathrm{~dB}$ gain. It is obvious that a TTI $=20$ milliseconds is the optimum choice: a further TTI increase gives no BER improvement but a gain loss of $0.6 \mathrm{~dB}$ at a BER of $10^{-3}$ and a loss of $2.5 \mathrm{~dB}$ at a BER of $3 \times 10^{-3}$. For $50 \mathrm{~km} / \mathrm{h}, 80$ milliseconds represents the ideal TTI choice in terms of performance and complexity. Particularly, at a BER of $10^{-3}$ performance gain of $6 \mathrm{~dB}, 2 \mathrm{~dB}$, and $0.8 \mathrm{~dB}$ is observed for TTI increase from 10 to 20 milliseconds, 20 to 40 milliseconds, and 40 to 80 milliseconds.

Figure 4 illustrates the BER performance for speeds of $100 \mathrm{~km} / \mathrm{h}$ and $300 \mathrm{~km} / \mathrm{h}$. For $100 \mathrm{~km} / \mathrm{h}$ at a BER of $10^{-3}$, a TTI of 20 milliseconds gives around $6 \mathrm{~dB}$ gain over

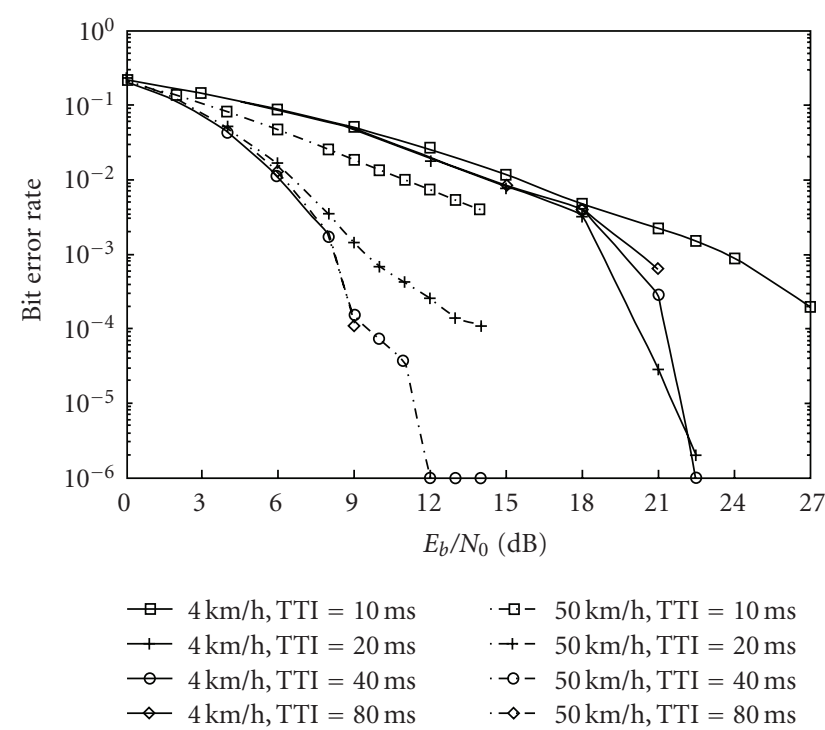

FIGURE 5: BER versus SNR for scenario 3 and $f_{d} T_{S}$ values 0.000017 and 0.00021 .

10 milliseconds, a TTI of 40 milliseconds gives $2.5 \mathrm{~dB}$ gain over 20 milliseconds, while a TTI of 80 milliseconds gives $0.25 \mathrm{~dB}$ gain over 40 milliseconds. For $300 \mathrm{~km} / \mathrm{h}$ terminal speed at a BER of $10^{-3}$, for a TTI increase from 10 to 20 , 20 to 40 , and 40 to 80 milliseconds, the corresponding improvements are $4 \mathrm{~dB}, 2 \mathrm{~dB}$, and $0.6 \mathrm{~dB}$. However, the optimum choice for both speeds is the maximum TTI value (80 milliseconds).

\subsection{Scenario 3: bit rate 144 kbps}

As can be observed from Figure 5 , for $4 \mathrm{~km} / \mathrm{h}$ mobile terminal speed, a TTI of 20 milliseconds is the optimum choice. Furthermore, at a BER of $10^{-3}$, the performance improvement using 20 milliseconds is $5 \mathrm{~dB}$ compared to 10 milliseconds. On the other hand, it is obvious that for TTI $=40$ milliseconds, there is a loss of $1 \mathrm{~dB}$ at a BER of $10^{-3}$, while for TTI $=80$ milliseconds there is a loss of $1.8 \mathrm{~dB}$ at the same BER compared to the optimum TTI value. For a terminal speed of $50 \mathrm{~km} / \mathrm{h}$, a TTI of 40 milliseconds represents the optimum solution. Consequently, the performance gain for a TTI increase from 10 to 20 milliseconds at a BER of $4 \times 10^{-3}$ is $6 \mathrm{~dB}$, while at a BER of $10^{-3}$ for a TTI increase from 20 to 40 milliseconds, the gain is $1.3 \mathrm{~dB}$.

Figure 6 illustrates the performance of the simulated system for terminal speeds $100 \mathrm{~km} / \mathrm{h}$ and $300 \mathrm{~km} / \mathrm{h}$. It is clear that for both speeds, the optimum value is 80 milliseconds. Among the three TTI values, a TTI of 20 milliseconds gives the highest improvement: at a BER of $10^{-2}$, the gain using 20 milliseconds compared to 10 milliseconds is $5 \mathrm{~dB}$, while using 40 milliseconds gives $2.2 \mathrm{~dB}$ gain compared to 20 milliseconds at a BER of $10^{-3}$. Also at a BER of $10^{-3}$, using 80 milliseconds gives $0.6 \mathrm{~dB}$ gain compared to 40 milliseconds. For a terminal speed of $300 \mathrm{~km} / \mathrm{h}$, again the highest performance improvement among the three possible TTI values (20, 40, 80 milliseconds) is 20 milliseconds: 


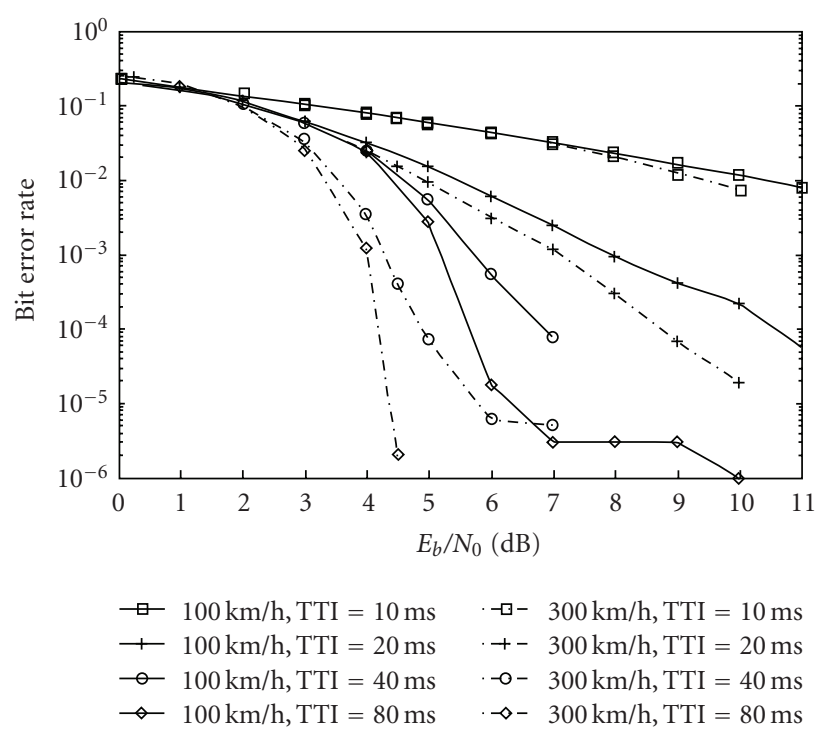

FIgURE 6: BER versus SNR for scenario 3 and $f_{d} T_{S}$ values 0.00042 and 0.00128 .

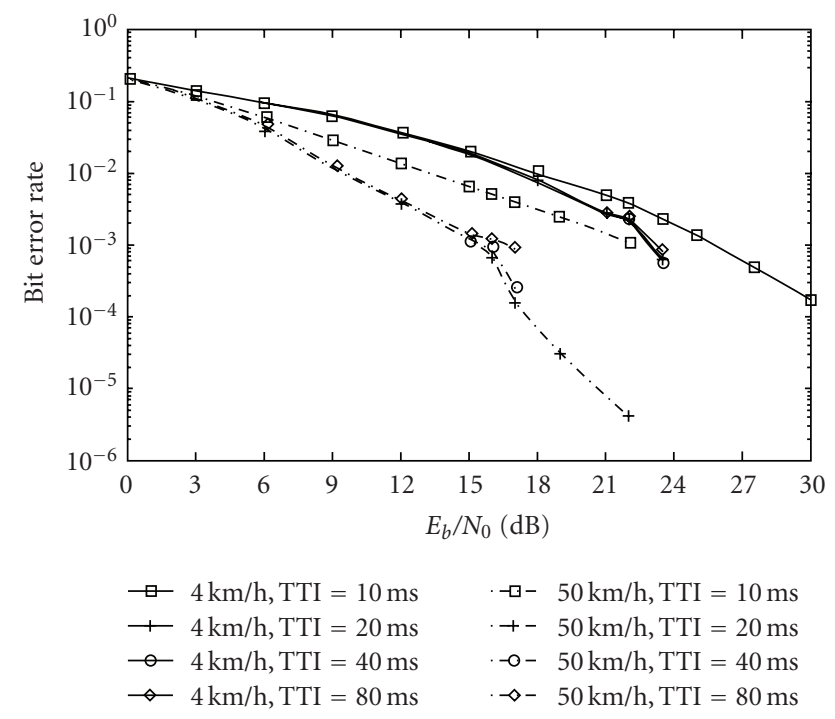

FIgURE 7: BER versus SNR for scenario 4 and $f_{d} T_{S}$ values 0.0000064 and 0.00008 .

at a BER of $10^{-2}$, the gain using TTI $=20$ milliseconds compared to 10 milliseconds is $4.3 \mathrm{~dB}$. At a BER of $10^{-3}$, using 40 milliseconds instead of 20 milliseconds gives a gain of $2.5 \mathrm{~dB}$, while using 80 milliseconds instead of 40 milliseconds gives a gain of $0.4 \mathrm{~dB}$.

\subsection{Scenario 4: bit rate 384 kbps}

Considering a large bit rate of $384 \mathrm{kbps}$, Figure 7 presents the BER of the simulated system for mobile terminal speeds of 4 and $50 \mathrm{~km} / \mathrm{h}$. As can be seen, 20 milliseconds is the optimum interleaver length for both speeds. For the first terminal speed and at a BER of $10^{-3}$, an increase of the TTI from 10 to 20 milliseconds gives a gain of $3 \mathrm{~dB}$. An increase of the

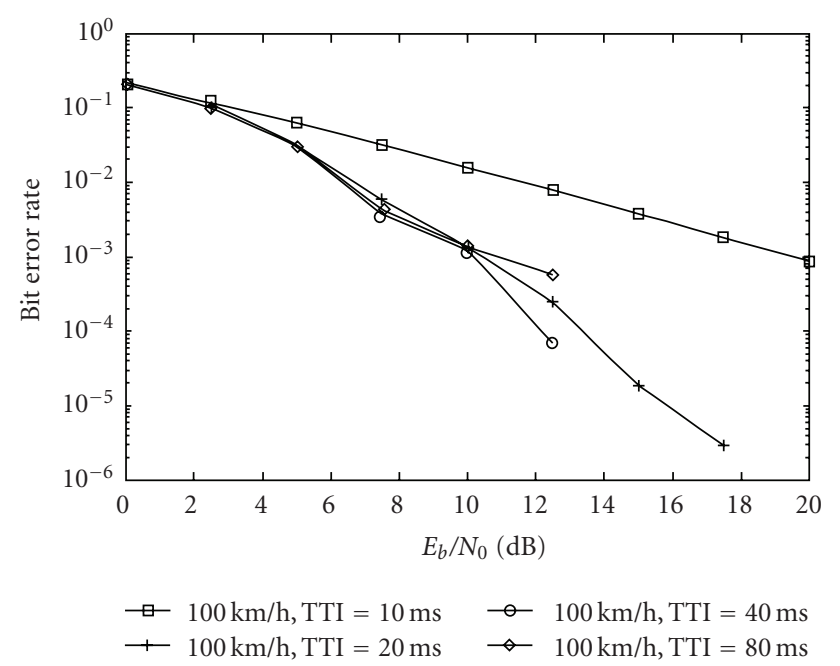

Figure 8: BER versus SNR for scenario 4 and $f_{d} T_{S}=0.00016$.

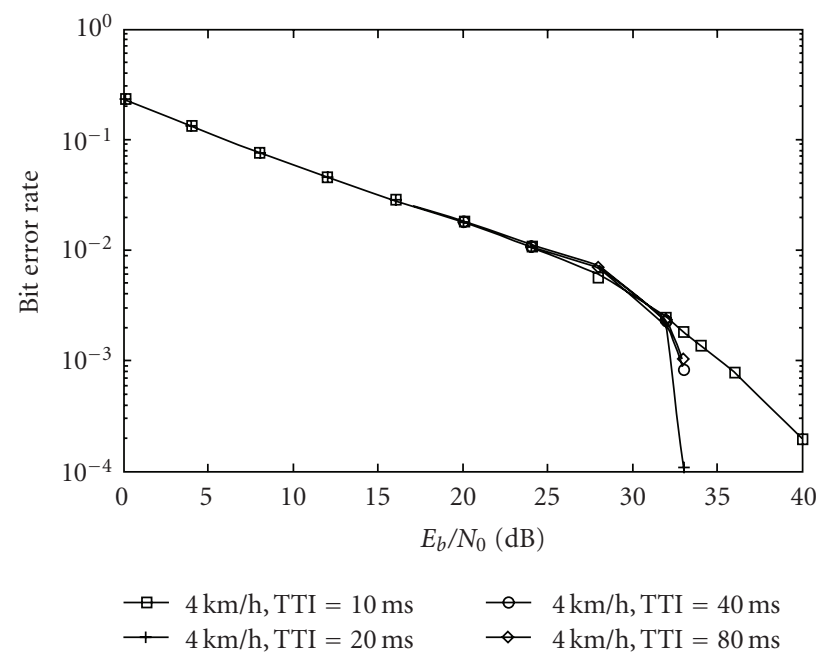

FIGURE 9: BER versus SNR for scenario 5 and $f_{d} T_{S}=0.0000012$.

TTI to 40 milliseconds at the same BER results in no performance loss, whereas a further increase ( 80 milliseconds) can cause $0.5 \mathrm{~dB}$ loss. For the second terminal speed and at a BER of $10^{-3}$, an increase of the TTI from 10 to 20 milliseconds gives a gain of $7 \mathrm{~dB}$. At the same BER for a TTI increase to 80 milliseconds, we observe performance losses of $1.5 \mathrm{~dB}$.

In Figure 8, the performance of the simulated system for a mobile terminal speed of $100 \mathrm{~km} / \mathrm{h}$ and bit rate $384 \mathrm{kbps}$ is evaluated. It is clear that 40 milliseconds is the optimum TTI value. Particularly, at a BER of $10^{-3}$, a performance gain of $9.5 \mathrm{~dB}$ and $0.8 \mathrm{~dB}$ is seen for a TTI increase from 10 to 20 and 20 to 40 milliseconds, respectively. Moreover, at the same BER, a performance loss of $1.5 \mathrm{~dB}$ is observed for further TTI increase (40 to 80 milliseconds). 
TABLE 4: Efficient TTI for different implementation scenarios and mobile terminal speeds based on BER performance.

\begin{tabular}{l|c|c|c|c|c}
\hline $\begin{array}{l}\text { Mobile terminal } \\
\text { speed }(\mathrm{km} / \mathrm{h})\end{array}$ & 1 & 2 & 3 & 4 & 5 \\
\cline { 2 - 6 } 4 & $20 \mathrm{~ms}$ & $20 \mathrm{~ms}$ & $20 \mathrm{~ms}$ & $20 \mathrm{~ms}$ & $20 \mathrm{~ms}$ \\
50 & $80 \mathrm{~ms}$ & $80 \mathrm{~ms}$ & $40 \mathrm{~ms}$ & $20 \mathrm{~ms}$ & - \\
100 & $80 \mathrm{~ms}$ & $80 \mathrm{~ms}$ & $80 \mathrm{~ms}$ & $40 \mathrm{~ms}$ & - \\
300 & $80 \mathrm{~ms}$ & $80 \mathrm{~ms}$ & $80 \mathrm{~ms}$ & - & - \\
\hline
\end{tabular}

\subsection{Scenario 5: bit rate 2 Mbps}

In Figure 9, the BER of the system for a mobile terminal speed of $4 \mathrm{~km} / \mathrm{h}$ and a high bit rate of $2 \mathrm{Mbps}$ is evaluated. A value of 20 milliseconds is shown to be the optimum TTI value. Particularly, at a BER of $10^{-3}$, a performance gain of $3 \mathrm{~dB}$ is seen for a TTI increase from 10 to 20 milliseconds. It is remarkable that at the same BER, a performance loss of $0.9 \mathrm{~dB}$ is observed for further TTI increase (20 to 40 milliseconds).

\section{CONCLUDING REMARKS}

Multimedia services represent the main novel services that $3 \mathrm{G}$ mobile communications can offer compared to 2G. Furthermore, high-data rates accommodate the introduction of multimedia services to $3 \mathrm{G}$ mobile phones. Thus, according to UMTS specifications, turbo codes represent the recently developed technology, which facilitates the efficient introduction of high-data rates in general and especially multimedia applications.

An efficient TTI choice in Rayleigh fading channels for different operating environments for UMTS $3 \mathrm{G}$ systems can reduce complexity and latency. These latency savings are very important, especially in the case of real-time multimedia services with tight delay constraints. Particularly, in Table 4, which summarises the simulation results presented in the previous section, the optimum TTI values are presented for the different scenarios and operating environments (mobile terminal speeds). Furthermore, our analysis shows that in terms of BER, an efficient TTI choice depends on bit rate and operating environment.

However, as can be seen in Table 4, for all five implementation scenarios considered, as a compromise between lowest BER and complexity (which means the best BER performance for the lowest TTI) and for a constant frame length of 5114 bits, a TTI $=20$ milliseconds (outer block interleaver with 2 columns) is recommended for indoor or low-range outdoor operating environment.

For an urban or suburban outdoor environment and for a terminal speed of $50 \mathrm{~km} / \mathrm{h}$, a TTI $=80$ milliseconds is optimum for relatively small bit rates like $28.8 \mathrm{kbps}$ and $64 \mathrm{kbps}$ (implementation scenarios 1 and 2), while as bit rate increases, the optimum TTI value decreases. Particularly, for increased bit rates like $144 \mathrm{kbps}$ and $384 \mathrm{kbps}$ (implementation scenarios 3 and 4), 40 milliseconds and 20 milliseconds are the suggested optimum values, respectively. For the same operating environment but a terminal speed of $100 \mathrm{~km} / \mathrm{h}$,
80 milliseconds is the right choice for bit rates $28.8 \mathrm{kbps}$, $64 \mathrm{kbps}$, and $144 \mathrm{kbps}$ (scenarios 1, 2, and 3). For bit rate $384 \mathrm{kbps}$ (scenario 4), 40 milliseconds should be used, while scenario 5 ( $2 \mathrm{Mbps}$ ) cannot be established. Again, the optimum TTI value decreases as bit rate increases, but after $144 \mathrm{kbps}$. As a general conclusion for this operating environment, we can say that for low bit rates, the optimum TTI value is the maximum value of 80 milliseconds. For increased bit rates, the optimum TTI value decreases. The decrease is faster for lower mobile terminal speeds. Moreover, in a rural outdoor operating environment, a TTI of 80 milliseconds is proposed for the first 3 scenarios, since scenarios 4 and 5 cannot be implemented.

Furthermore, our simulation results also show that choosing higher TTI than the optimum value causes negative effects on BER. Particularly, in indoor or low-range outdoor operating environment for all bit rates, the choice of a TTI value which is larger than the optimum one can cause up to $1.8 \mathrm{~dB}$ BER loss. This value occurs at a medium bit rate of $144 \mathrm{kbps}$. The same effect is also seen at high-bit rates (384 kbps) in urban or suburban outdoor operating environment with a performance loss of $1.5 \mathrm{~dB}$. However, as in [17] for block codes, the optimum choice of block interleaver length in different fading environments is also essential for turbo codes. Finally, the simulation results show that a TTI increase from 10 to 20 milliseconds gives the highest performance gain compared to the other TTI increases (20 to 40 and 40 to 80 milliseconds) for all bit rates considered. On the other hand, the lowest gain is given by a TTI increase from 40 to 80 milliseconds. This is what we expect, since performance gain decreases as the number of columns of outer block interleaver increases $[15,16]$.

\section{REFERENCES}

[1] C. Berrou and A. Glavieux, "Near optimum error correcting coding and decoding: turbo-codes," IEEE Transactions on Communications, vol. 44, no. 9, pp. 1261-1271, 1996.

[2] S. S. Pietrobon, "Implementation and performance of a turbo/MAP decoder," International Journal of Satellite Communications, vol. 16, no. 1, pp. 23-46, 1998.

[3] P. Robertson, E. Villebrun, and P. Hoeher, "A comparison of optimal and sub-optimal MAP decoding algorithms operating in the log domain," in Proceedings of the IEEE International Conference on Communications (ICC '95), vol. 2, pp. 10091013, Seattle, Wash, USA, June 1995.

[4] C. Chaikalis and J. M. Noras, "Reconfigurable turbo decoding for 3G applications," Signal Processing, vol. 84, no. 10, pp. 1957-1972, 2004. 
[5] C. Chaikalis, "Reconfiguration aspects and a reconfigurable outer block interleaver for 3G applications," Wireless Personal Communications, vol. 41, no. 1, pp. 77-97, 2007.

[6] C. Chaikalis, Reconfigurable structures for turbo codes in $3 \mathrm{G} \mathrm{mo-}$ bile radio transceivers, $\mathrm{Ph}$.D. thesis, Department of Electronics \& Telecommunications, University of Bradford, Bradford, UK, 2003.

[7] J. P. Woodard and L. Hanzo, "Comparative study of turbo decoding techniques: an overview," IEEE Transactions on Vehicular Technology, vol. 49, no. 6, pp. 2208-2233, 2000.

[8] H. Holma and A. Toskala, Eds., WCDMA for UMTS: Radio Access for Third Generation Mobile Communications, John Wiley \& Sons, Chichester, UK, 2000.

[9] F. Muratore, Ed., UMTS: Mobile Communications for The Future, John Wiley \& Sons, Chichester, UK, 2001.

[10] S. Kotsopoulos and D. Lymberopoulos, "A new medical data management concept in a hybrid cellular mobile radio communication network," in Proceedings of the IEEE Global Telecommunications Conference (GLOBECOM '91), vol. 1, pp. 674-680, Phoenix, Ariz, USA, December 1991.

[11] S. Kotsopoulos and D. Lymberopoulos, "Communication protocols and on-board processor for a new national scale private mobile radio service," in Proceedings of IEEE International Conference on Selected Topics in Wireless Communications, pp. 147-150, Vancouver, BC, Canada, June 1992.

[12] S. Bouzouki, S. Kotsopoulos, G. Karagiannidis, K. Chassomeris, and D. Lymberopoulos, "On optimal cell planning: case study for a DCS 1800 system," International Journal of Communication Systems, vol. 14, no. 9, pp. 857-870, 2001.

[13] K. Ioannou, I. Panoutsopoulos, S. Koubias, and S. Kotsopoulos, "A new dynamic channel management scheme to increase the performance index of cellular networks," IEE Electronics Letters, vol. 40, no. 12, pp. 744-746, 2004.

[14] E. K. Hall and S. G. Wilson, "Design and analysis of turbo codes on Rayleigh fading channels," IEEE Journal on Selected Areas in Communications, vol. 16, no. 2, pp. 160-174, 1998.

[15] K. Tang, P. H. Siegel, and L. B. Milstein, "On the performance of turbo coding for the land mobile channel with delay constraints," in Proceedings of the 33rd Asilomar Conference on Signals, Systems and Computers (ACSSC '99), vol. 2, pp. 16591664, Pacific Grove, Calif, USA, October 1999.

[16] F. Poppe, D. De Vleeschauwer, and G. H. Petit, "Guaranteeing quality of service to packetised voice over the UMT Sair interface," in IEEE 8th International Workshop on Quality of Service (IWQOS '00), pp. 85-91, Pittsburgh, Pa, USA, May-July 2000.

[17] K. I. Chan and J. C.-I. Chuang, "Required interleaving depth in Rayleigh fading channels," in Proceedings of the IEEE Global Telecommunications Conference (GLOBECOM '96), vol. 2, pp. 1417-1421, London, UK, November 1996.

[18] M. Pätzold, U. Killat, F. Laue, and Y. Li, "On the statistical properties of deterministic simulation models for mobile fading channels," IEEE Transactions on Vehicular Technology, vol. 47, no. 1, pp. 254-269, 1998.

[19] 3GPP TS 25.212 V3.9.0, "Multiplexing and channel coding (FDD)," Release 1999, March 2002.

[20] 3GPP TS 25.222 V3.8.0, "Multiplexing and channel coding (TDD)," Release 1999, March 2002.

[21] 3GPP TR 25.944 V3.5.0, "Channel coding and multiplexing examples," Release 1999, June 2001.

[22] 3GPP TS 25.201 V3.3.0, "Physical layer-general description," Release 1999, March 2002. 

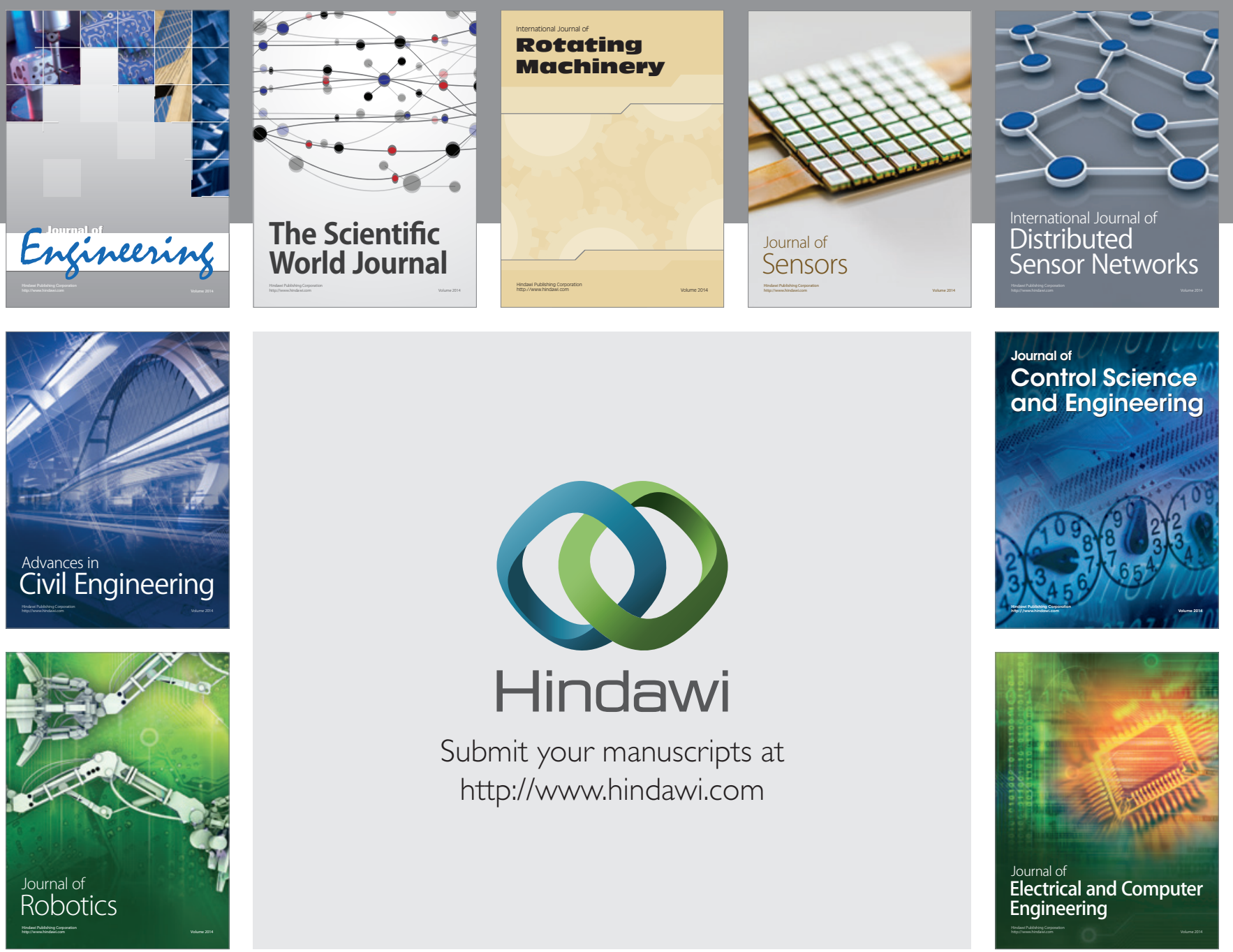

Submit your manuscripts at

http://www.hindawi.com
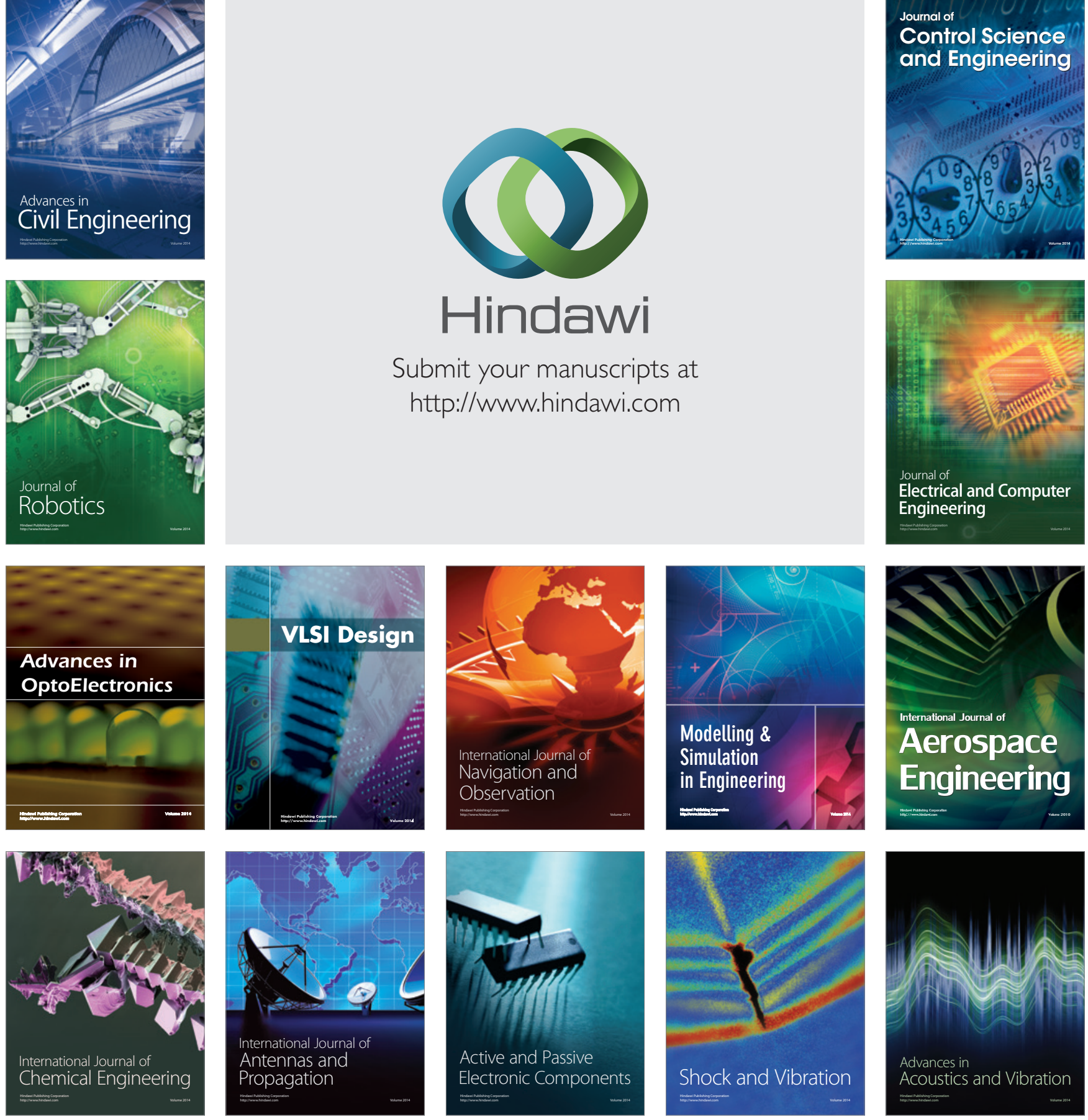